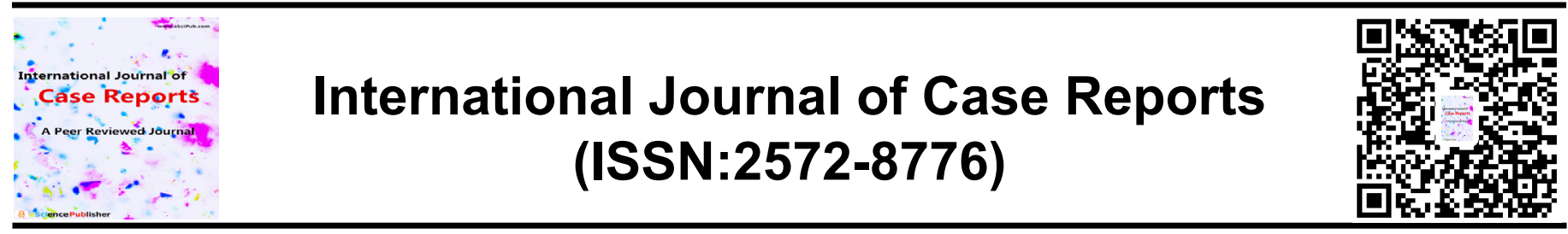

\title{
Ptosis resolved after cataract surgery - A case report
}

\author{
Ming Chen, MD, MSc, FACS
}

University of Hawaii.

\section{ABSTRACT}

\section{Introduction}

${ }^{*}$ Correspondence to Author:

It is well known in the literature that adult ptosis can be induced Ming Chen, MD, MSc, FACS or worsen by cataract surgery even with modern sutureless clear cornea phacoemulsification. ${ }^{[1,2,3,4,5,6,7,8]}$ We are presenting this unusual case was the opposite. The ptosis resolved after cataract surgery. A possible effect from the successful management of dry eye disease before cataract surgery is explained.

University of Hawaii.

Drooping of the unilateral upper eyelid is unilateral ptosis. Ptosis may be myogenic, neurogenic, and aponeurotic from healthy aging, injury, eye diseases. ${ }^{[9,10]}$ Ptosis also can be associated with hard contact lens wear, immunological, degenerative, or hereditary disorders, tumors, or infections. ${ }^{[7]}$

Patients with ptosis often present with a lower position of the upper eyelid, complain of a tired appearance, and defect in their superior visual field even blurred vision. The levator muscle controls the position of the eye lid and is innervated by the How to cite this article:

Ming Chen, MD, MSc, FACS. Ptosis resolved after cataract surgery - $A$ case report. International Journal of Case Reports, 2020; 4:157. oculomotor nerve. The Mueller muscle also controls partially by providing sympathetic innervation. The deficiency of these two muscles may cause ptosis. ${ }^{[11]}$

Hering's law of motor correspondence of a ptosis lid can affect the contralateral lid position ${ }^{[5,7]}$ as the levator muscles work in synchrony with each other. Therefore, unilateral ptosis can 
induce a retraction of the contralateral lid or a pseudo retraction. When ptosis is causing visual field defects, the innervation input to both eyelids will increase to reduce the ptosis. There will be a compensatory elevation of eye brow to reduce the amount of ptosis. [11] These phenomena need to be considered in the evaluation of unilateral ptosis for surgery. Another essential examination, such as Margin Reflex Distance 1 (MRD1), is the crucial measurement for ptosis besides levator function and palpebral fissure. MRD1 is the distance from the margin of the upper lid to the central corneal reflex (normal is between 4.0-4.5 mm). ${ }^{[11]}$

\section{Case presentation}

Sixty-six years old Chinese female presented in the clinic complained of dry eye, blurred vision, and drooping lid over the right eye for six months. She indicated that she was diagnosed with a cataract in Hong Kong and was recommended to have cataract surgery. She had no history of myasthenia gravis or thyroid disease, as well as other causes leading to the ptosis. She has type 2 Diabetics with reasonable control and no retinopathy. Her history revealed having a dry eye since 2005 and cataract since 2018. She did not wear any contact lens before. Her vision over the right eye was 20/40 with the best correction of $1.25+0.25 \times 134$, tested with the glare; vision dropped down to 20/200; the left eye was 20/30. Intraocular pressure was normal. Pupils were equal and reactive to light. There was full eye movement; both eyes moved in all directions without strabismus.

On the examination, right eye appeared to have recent ptosis that showed MRD1=1 (Figure 1), which responded to $2.5 \%$ Neosynephrine eye drop and improved to MRD1=3 and the levator muscle function appeared to be normal.

Figure 1: Before cataract surgery

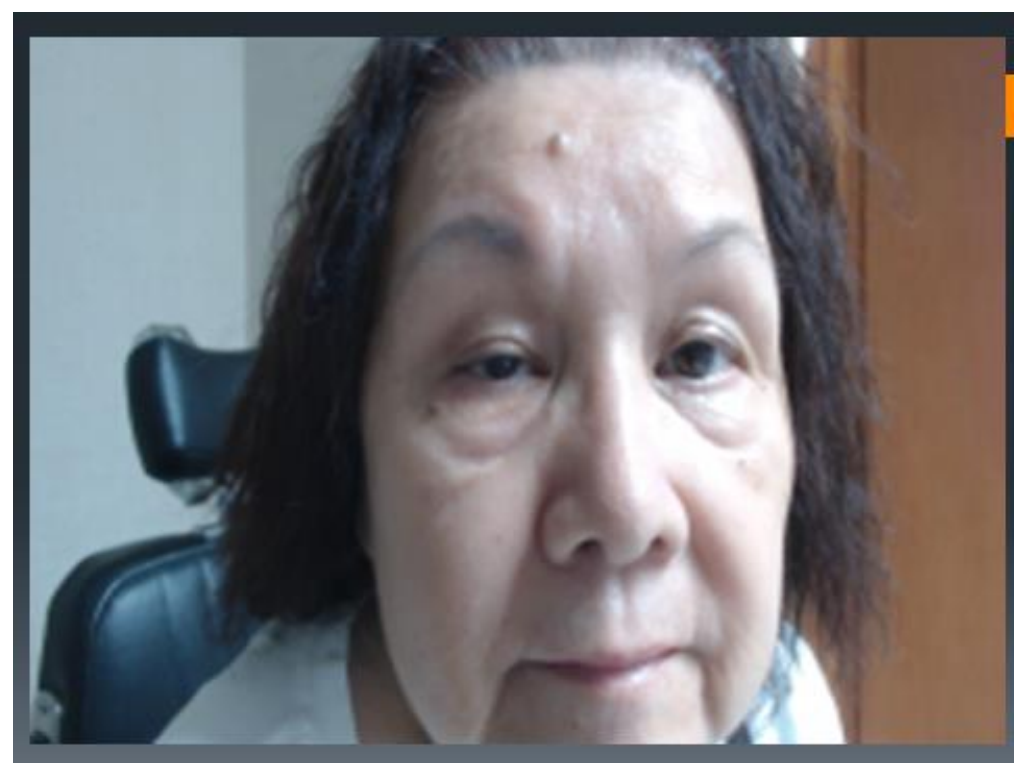

The Hering's law was not evident as the contralateral eye lid did not shows retraction. Under the slit lamp examination, the cornea shows level 3 of dry eye disease (graded with International Dry Eye Workshop 1 (DREW 1) ${ }^{[2]}$ with diffuse punctate staining. The endothelium appeared normal. There was bilateral cortical cataract, and the right eye cataract was denser. Fundus examinations of both eyes were normal.

The diagnosis was a recent on-set of unilateral 
adult ptosis that may be secondary to dry eye disease and aggravated by a significant cataract.

The management plan was to treat dry eye disease first, follow with cataract surgery than possible ptosis repair with the modified sutureless Fasanella-Servat procedure if ptosis persists after cataract surgery. ${ }^{[13]}$

The patient was put on artificial tears drop four times a day and the Erythromycin ointment before bedtime. All four eye's punctum was closed with cautery. After two weeks, as the dry eye disease improved, routine cataract surgery using phacoemulsification under topical anesthesia with intraocular lens implant was perfor- med carefully without complication through a temporal clear cornea incision. The post-operative medications included $1 \%$ prednisone ophthalmic solution and Ocuflox eye drop four times a day, Prolensa eye drop once a day for one week, then follow the sliding scale to reduce dose and to complete in one month.

The one week post-operative examination showed her right ptosis has resolved considerably. (Figure 2) Vision was 20/25 with the refraction of $-0.50+0.50 \times 17$. The cornea staining was not detectable and had no complain of dry eye. The rest of the examinations were normal. Onemonth post-operative exam was the same.

Figure 2: One week after cataract surgery

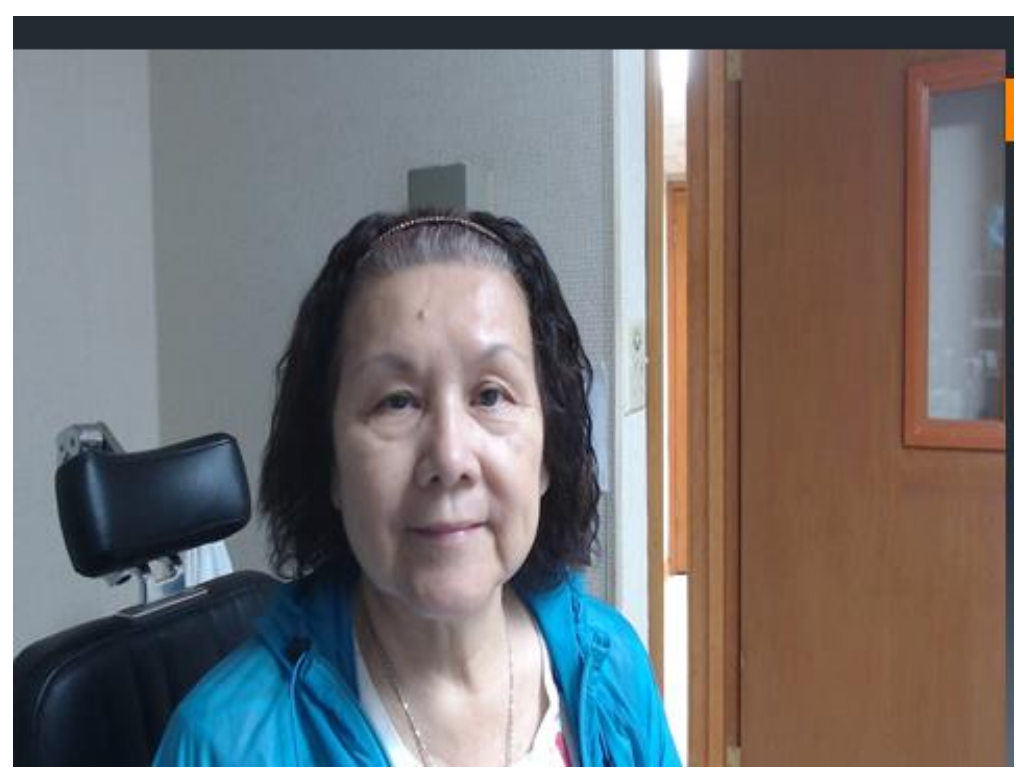

\section{Discussion}

Older literature demonstrated cataract surgery can cause or worsen ptosis for various reasons. $[1,2,3,4,5,6,7,8]$ Those days cataract was done using bridal suture over superior rectus muscle with larger wound. Trauma to the levator muscle and inflammation to the eye was frequent. Recent report with modern sutureless clear cornea phacoemulsification still had about $3 \%$ incidence. ${ }^{[3]}$ Dre eye treatment was not popular during those days and in some country currently. These may be the reasons that incidence of post-cataract surgery ptosis was high. In this case, as other etiologic reasons causing ptosis were ruled out, this recent unilateral adult ptosis may be induced by the dry eye disease and aggravated by a significant cataract. Therefore, after treatment of dry eye and modern cataract surgery the ptosis resolved. Parsa et al reported in1996, suggested waiting 6-month before repairing post-cataract 
surgery ptosis as he presented a case that developed overcorrection of a post cataract surgery ptosis. ${ }^{8}$ Ptosis developed before or after cataract surgery should be carefully evaluated for cornea staining from dry eye disease.

\section{Conclusion}

The recent onset unilateral adult ptosis was resolved after cataract surgery and dry eye treatment in this case. Uneventful cataract surgery and dry eye treatment may improve adult ptosis.

\section{References}

[1] Kaplan LJ, Jaffee NS.Clayman HM, Ptosis and cataract surgery. A multivariant computer analysis of a prospective study, Ophthalmology 1985; 92(2):237-242

[2] Puvanachandra N, Hustler A,Seah LL, Tyers AG The incidence of ptosis following extracapsular and phacoemulcification surgery: comparison of two prospective studies and review of the literature. Orbit 2010; 29(6):321-323

[3] Kashkouli MB, Abdolalizadeh P, Es'Haghi A, et $\mathrm{Al}$, Postoperative blepharoptosis after modern phacoemulsification procedure, American journal of ophthalmology, 2020: 213:17-23

[4] Loeffler M, Solomon L, Renaud M. Postcataract extraction ptosis: Effect of the bridle suture. J Cataract Refract Surg 1990;16:501-540.

[5] Patel JI, Blount M, Jones C. Surgical blepharoptosis-the bridle suture factor, Eye 2020;16: 535-537.

[6] Linberg JV, McDonald MB, Safir A. Googe JM. Ptosis following radial keratotomy, Ophthalmol 1986;93:1509-1512

[7] Parsa FD, Wolff DR, Parsa NN, Elahi aE E Upper eyelid ptosis repair after cataract extraction and the importance of Hering's test., Plast Re-constr Surg. 2001 Nov;108(6):152736; discus-sion 1537-8.

[8] Parsa FD, Roach JM. A case report on spontaneous return of levator functions following post cataract blepharoplasty repair. Ann Plast Surg 1996;37: 638-649

[9] Finsterer J. Ptosis: causes, presentation, and management Aesthetic Plast Surg. 2003 May-
-Jun;27(3):193-204. Epub 2003 Aug 21

[10] Watanabe A, Araki B, Noso K Kakizaki H ,Kinoshita S, Histopathology of Blepharoptosis In-duced by Prolonged Hard Contact Lens Wear,https://doi.org/10.1016/j.ajo.2006.01.032

[11] Zoumalan C; Lisman R, Evaluation and Management of Unilateral Ptosis and Avoiding Contralateral Ptosis, Aesthetic Surgery Journal 30(3) $320-328$ @ 2010

[12] Lemp M, et Al, DEWS Definition and Classification, the Ocular Surface / April 2007, Vol. 5, No. 2

[13] Chen M, MODIFIED SUTURELESS OPERATION FOR MILD BLEPHAROPTOSIS REPAIR International Journal of Academic Research and Reflection Vol. 5, No. 2, 2017 ISSN 23090405 